\title{
'|||||||||||||||||||||||||||||||||||||||||||||||||||||||||||||||||.
}

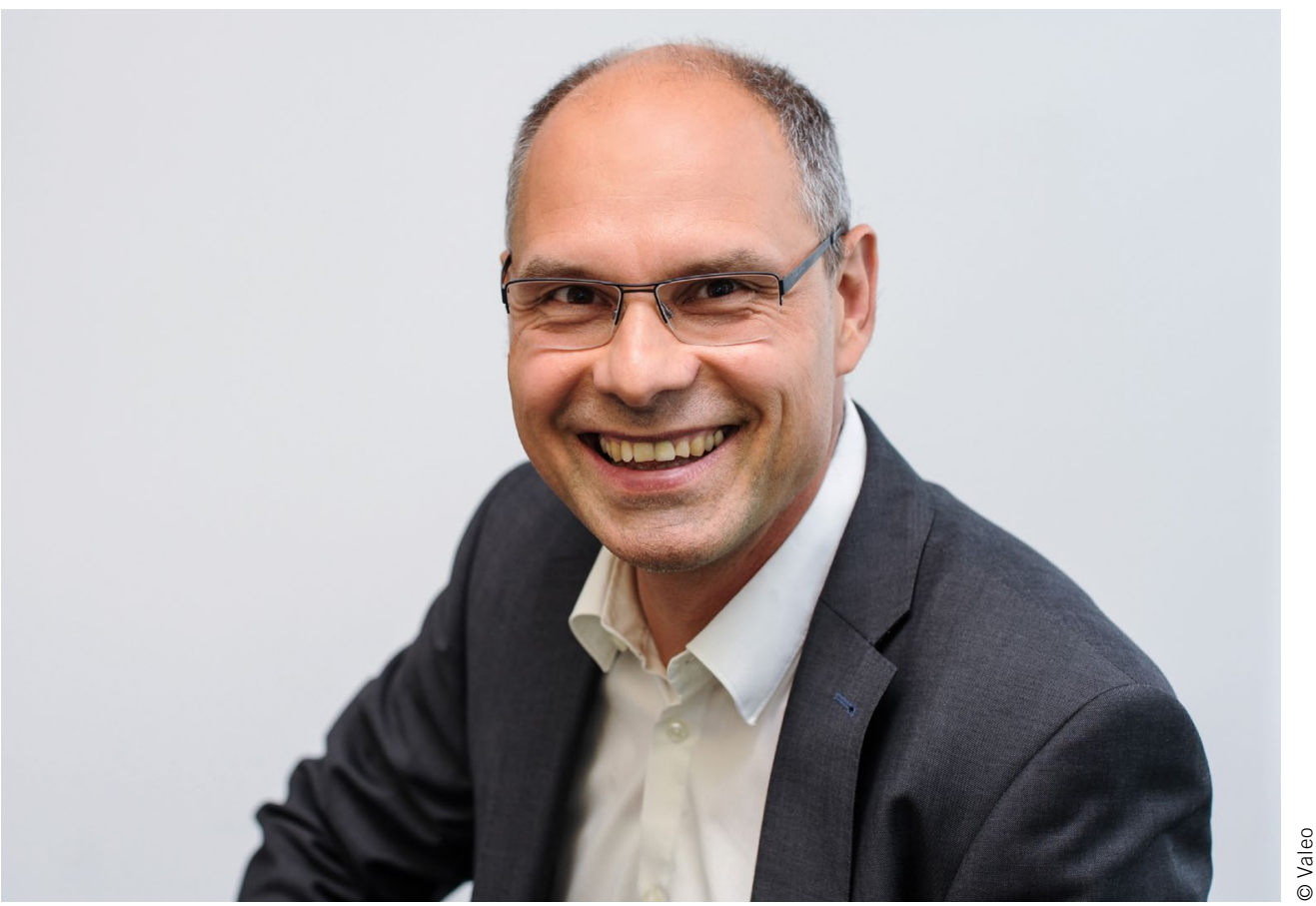

Joachim Mathes

Chief Technology Officer of Valeo Comfort \& Driving Assistance Systems, Member of the ATZ Scientific Advisory Board

\section{Stylish Living on Four Wheels}

Until recently, the driver-oriented cockpit was considered the pinnacle of desirable design, with the driver taking center stage. But now the inside of the car is being transformed into a lounge for all the occupants. Driving is fast becoming a background activity and individual comfort and an atmosphere of well-being for the passengers in all the seats are increasing in importance. The car will live up to the high standards demanded of it by recognizing the occupants, analyzing their mood and state of health and learning their various requirements and preferences. The design of the interior and digital continuity - in other words, end-to-end connectivity - are now among the most important purchase criteria, in particular in China. But with all these distractions, who is still paying attention to the traffic?

Today's automated systems focus on avoiding accidents and assisting the driver. Even in partially automated cars, drivers can turn their attention temporarily to other activities. In Japan, SAE level 3 production models are already on the roads and these will be followed by cars from other manufacturers in the next twelve months.

The interior of cars is being designed to provide suitable projection surfaces, such as the area under the windshield or even the windshield itself, which can be turned into a display where reality and the virtual world are superimposed. The challenge for developers is to display information relating to the vehicle's current situation only when and where this makes sense.
The other surfaces inside the car are also undergoing a similar transformation. On the one hand, there is a desire for natural materials to be used as a visible indication of sustainability and the need to feel secure. On the other hand, these surfaces have to fulfill a wide range of functions from interaction via touch and gestures to providing heating. All of these functions will remain largely invisible, because nothing will be allowed to disturb the simple aesthetics of the design.

In some respects, the interiors of modern cars are more comfortable and smarter than many homes. This makes the idea of turning the car into a living space on four wheels just as logical as it is radical. At the Shanghai Motor Show 2021, Xiaopeng had its new P5 sedan on its stand fitted out as a mobile home with optional cinema mode and double bed. As most cars spend more than $90 \%$ of the time parked up somewhere and in future will have large batteries that will make them energy-independent during these periods, many "drivers" may be happy to use their vehicles as a living room.

This calls into question our entire existing mobility model, but it also demonstrates that we are in the middle of a very exciting phase of change and that the major markets in Asia and, in particular, in China will play an important role in determining the future direction of the mobility industry. We need to take a global approach to the interior of the future so that we can make life on board comfortable and safe for car occupants. 